\title{
Incremental Versus Conventional Hemodialysis for Preservation of Residual Kidney Function in Patients on Regular Hemodialysis
}

\author{
Hosam Ezzat Ebrahim, ${ }^{1, *}$ MSc; Emad Allam Mohamed ${ }^{2}$ MD ; Mohamed Ahmed El Sayed Ahmed ${ }^{2}$ MD \\ Osama Hassan Bakheet ${ }^{2}$ MD
}

\author{
*Corresponding Author: \\ Hosam Ezzat Ebrahim \\ cristiano9966@gmail.com
}

Received for publication October 31, 2020; Accepted January 3, 2021; Published online January 3, 2021.

\section{Copyright 2020 The Authors} published by Al-Azhar University, Faculty of Medicine, Cairo, Egypt. All rights reserved. This an openaccess article distributed under the legal terms, where it is permissible to download and share the work provided it is properly cited. The work cannot be changed in anyway or used commercially.

doi: $10.21608 /$ aimj.2021.45156.1338

${ }^{1}$ Clinical pathology Department, Military Medical Academy, Cairo, Egypt.

${ }^{2}$ Internal medicine and nephrology Department, Faculty of Medicine, Al-Azhar University, Cairo, Egypt.

Disclosure: The authors have no financial interest to declare in relation to the content of this article. The Article Processing Charge was paid for by the authors.

Authorship: All authors have a substantial contribution to the article.

\begin{abstract}
Background: Incremental hemodialysis (HD) has several potential advantages for patients, physicians and health care systems. In incident HD patients, conservation of residual kidney function "RKF" is significant and is correlated with several advantages, including survival of patient, improved quality of life, improved overall nutritional status and less anemia. The aim of this work was to evaluate incremental hemodialysis (twice/week) compared with conventional dialysis (thrice/week) for the conservation of the residual kidneyfunction among patients initiating regular hemodialysis.

Aim of work: The primary outcome of the study was an evaluation of incremental hemodialysis (twice/week) compared with conventional dialysis (thrice/week) for the conservation of the residual kidney function among patients initiating regular hemodialysis.

Patient and Methods: We assigned 40 patients with chronic kidney disease stage V D who early started hemodialysis into 2 groups: Group 1: 20 patients underwent incremental hemodialysis (twice/week), and Group 2: 20 patients underwent conventional hemodialysis (thrice/week). All patients followed for laboratory findings of (complete blood picture, creatinine, urea, calcium, albumin, phosphorus, parathyroid hormone, alkaline phosphatase, glomerular filtration rate). Also, Kt/v at start and every month was done. Clinical outcome measures included; mortality, cardiovascular outcome, hospital admission and dialysis complications.

Results: In the studied population, the mean age was $(45.95 \pm 2.97)$ years. Regarding sex $60 \%$ were males and $40 \%$ were females. Regarding outcome data, $(20 \%)$ of patients had hypotension, $(15 \%)$ had hospital admission, while nobody suffered mortality. Comparative study among the two groups showed a substantial decrease in the incidence of hypotension in the incremental group $(40 \%)$ relative to the conventional group $(10 \%)(\mathrm{p}=0.03)$.

Conclusion: To conclude, our research investigates the correlation among the frequency of HD treatment and survival of patient. In line with recent literature, our results show that some selected patients with acceptable RKF, sufficient control of interdialytic gaining weight, and low or moderate burden of comorbid disease $(\mathrm{CCI}<5)$ in an incident HD population, an HD incremental approach may be deemed as an appropriate alternative to conventional thrice-weekly HD.
\end{abstract}

Keywords: Incremental dialysis; Conventional dialysis; Residual Kidney Function. 
significant and is correlated with many advantages, like patient longevity, improved quality of life, enhanced overall dietary status and decreased anemia (The Importance of Residual Kidney Function, see above). The "longevity of vascular access" associated with less regular arteriovenous fistula or graft cannulations are another advantage of incremental HD. In an examination from the FHN study, more frequent HD decreased the composite endpoint of vascular access loss, repair or accessrelated hospitalization. The chance of a first access event was $76 \%$ higher with regular HD than with conventional $\mathrm{HD}^{5}$.

For less frequent HD therapy regimens, economic advantages also need to be considered.Conventional thrice-weekly HD treatments cost around $\$ 89,000$ per person per year in the United States, with a gross annual cost of $\$ 42$ billion ( $\$ 34$ billion covered by Medicare, the rest by , private insurance, Medicaid or out-of-pocket payments) ${ }^{6}$

\section{PATIENTS AND MATERIALS}

We assigned 40 patients with chronic kidney disease stage V D who early started hemodialysis into 2 groups:Group 1: 20 patients underwent incremental hemodialysis (twice/week).and Group 2: 20 patients underwent conventional hemodialysis (thrice/week).

During 6 months in Nephrology department, Military Hospitals (AL-maadi, kobry AL-koba and air force military hospital).

All patients will be subjected to:History \& complete physical examination, Routine laboratory investigations: $\mathrm{CBC}$, liver and kidney function Calcium, phosphorus, intact parathyroid hormone GFR and KT/V. Clinical outcome

Measures:Mortality,Cardiovascularoutcome,Hospital admission and Dialysis complications,

Statistical analysis:

Using MedCalc ver. 18.2.1 (MedCalc, Ostend, Belgium), data input, processing and statistical analysis have been conducted out. Significance tests were used (Mann-Whitney's, Chi square, tests, factorial ANOVA, logistic regression analysis, and analysis of the ROC Curve). Data were supplied and appropriate analysis was carried out as per type of data obtained for each variable (parametric and nonparametric). P-values of lower than $0.05(5 \%)$ were deemed statistically important.

\section{RESULTS}

In the studied population, the mean age of all patients was $(45.95 \pm 2.97)$ years, and the average UOP was $(1124.3 \pm 337.2) \mathrm{ml} /$ day. Regarding gender of the patients, the majority $(60 \%)$ of patients were males; while $(40 \%)$ were females, with $(65 \%)$ had DM, and (75\%) had HTN.

Regarding outcome data, (20\%) of patients had hypotension, (15\%) had hospital admission, while nobody suffered mortality.

Comparative studies:The $40 \mathrm{CKD}$ patients were split into two separate groups accordingly to the dialysis
technique:Conventional group (20 patients) and Incremental group (20 patients).

Non-significant differences in all basic clinical data were observed in a comparative analysis among the two groups $(\mathrm{p}>0.05)$.

Non-significant differences in all baseline laboratory data were observed in the comparative analysis among the two groups $(\mathrm{p}>0.05)$.

ROC curve analysis to predict patients (6-months) improvement (Table 1, Fig. 1 - 4): By using ROCcurve analysis, Incremental dialysis technique expected patients with hemoglobin improvement, with excellent $(93 \%)$ accuracy, sensitivity $=75 \%$ and specificity $=100 \%(\mathrm{p}<0.05)$.

By using ROC-curve analysis, Incremental dialysis technique predicted patients with platelets improvement, with fair $(70 \%)$ accuracy, sensitivity= $55 \%$ and specificity $=100 \%(\mathrm{p}<0.05)$.

By using ROC-curve analysis, Incremental dialysis technique predicted patients with GFR improvement, with perfect $(100 \%)$ accuracy, sensitivity $=100 \%$ and specificity $=100 \%(\mathrm{p}<0.05)$

By using ROC-curve analysis, Incremental dialysis technique predicted patients with uric acid improvement, with fair $(70 \%)$ accuracy, sensitivity= $50 \%$ and specificity $=90 \%(\mathrm{p}<0.05)$

By using ROC-curve analysis, Incremental dialysis technique expected patients with $\mathrm{Ca}$ improvement, with excellent $(96 \%)$ accuracy, sensitivity $=100 \%$ and specificity $=80 \%(\mathrm{p}<0.05)$.

By using ROC-curve analysis, Incremental dialysis technique expected patients with $\mathrm{Ph}$ improvement, with excellent $(94 \%)$ accuracy, sensitivity $=85 \%$ and specificity $=90 \%(\mathrm{p}<0.05)$.

By using ROC-curve analysis, Incremental dialysis technique expected patients with PTH improvement, with excellent $(91 \%)$ accuracy, sensitivity $=80 \%$ and specificity $=90 \%(\mathrm{p}<0.05)$.

By using ROC-curve analysis, Incremental dialysis technique predicted patients with albumin improvement, with fair $(79 \%)$ accuracy, sensitivity= $55 \%$ and specificity $=90 \%(\mathrm{p}<0.05)$.

By using ROC-curve analysis, Incremental dialysis technique predicted patients with $\mathrm{Kt} / \mathrm{V}$ improvement, with good (89\%) accuracy, sensitivity $=60 \%$ and specificity $=100 \%(\mathrm{p}<0.05)$

By using ROC-curve analysis, Incremental dialysis technique showed non-significant predictive values regarding TLC, creatinine, urea, $\mathrm{Na}$ and $\mathrm{K}$ improvements $(p>0.05)$. 


\begin{tabular}{|c|c|c|c|c|c|}
\hline Variable & AUC & SE & $\begin{array}{c}\text { Sensitivity } \\
(\%)\end{array}$ & $\begin{array}{c}\text { Specificity } \\
(\%)\end{array}$ & P value \\
\hline $\mathrm{Hb}$ & 0.930 & 0.038 & 75 & 100 & $<0.0001^{* *}$ \\
\hline PLT & 0.700 & 0.087 & 55 & 100 & $0.022^{*}$ \\
\hline TLC & 0.585 & 0.093 & 65 & 60 & 0.3652 \\
\hline $\begin{array}{c}\text { Creat. } \\
\text { (post- } \\
\text { dialysis })\end{array}$ & 0.660 & 0.090 & 90 & 60 & 0.0781 \\
\hline $\begin{array}{c}\text { Urea } \\
\text { (post- } \\
\text { dialysis })\end{array}$ & 0.600 & 0.094 & 100 & 40 & 0.2882 \\
\hline GFR & 1.000 & 0 & 100 & 100 & $<0.0001^{* *}$ \\
\hline $\begin{array}{c}\text { Uric } \\
\text { acid }\end{array}$ & 0.703 & 0.083 & 50 & 90 & $0.015^{*}$ \\
\hline $\mathrm{Na}$ & 0.623 & 0.096 & 40 & 100 & 0.2053 \\
\hline $\mathrm{K}$ & 0.588 & 0.091 & 90 & 30 & 0.3399 \\
\hline $\mathrm{Ca}$ & 0.968 & 0.023 & 100 & 80 & $<0.0001^{* *}$ \\
\hline $\mathrm{Ph}$ & 0.941 & 0.033 & 85 & 90 & $<0.0001^{* *}$ \\
\hline $\mathrm{PTH}$ & 0.915 & 0.043 & 80 & 90 & $<0.0001^{* *}$ \\
\hline $\mathrm{Alb}$. & 0.790 & 0.072 & 55 & 90 & $0.0001^{* *}$ \\
\hline $\mathrm{Kt} / \mathrm{V}$ & 0.895 & 0.048 & 60 & 100 & $<0.0001^{* *}$ \\
\hline $\mathrm{ROC}(\mathrm{R}$ & & & & & \\
\hline
\end{tabular}

Fig.2: ROC curve of GFR and uric acid (Incremental dialysis technique).

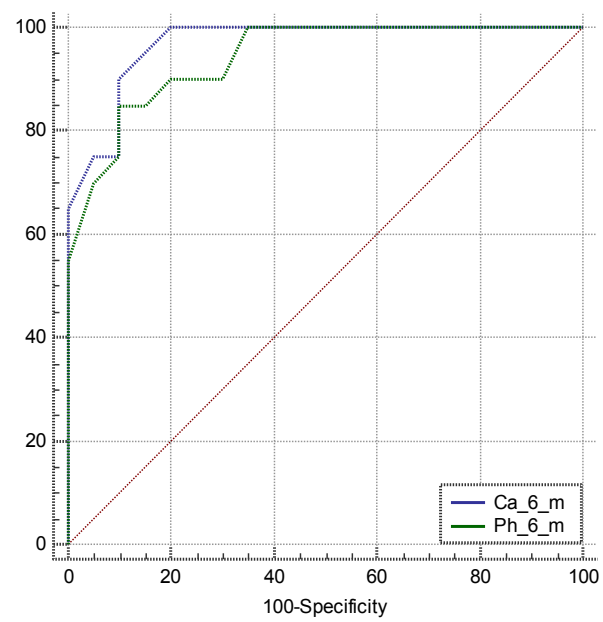

Fig.3: ROC curve of $\mathrm{Ca}$ and $\mathrm{Ph}$ (Incremental dialysis technique).

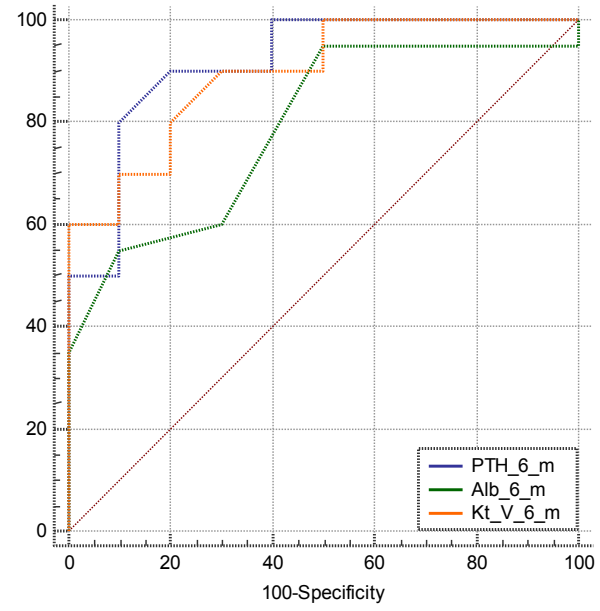

Fig.4: ROC curve of PTH, albumin and $\mathrm{Kt} / \mathrm{V}$ (Incremental dialysis technique).

\begin{tabular}{|c|c|c|c|c|}
\hline \multicolumn{2}{|c|}{ Variable } & $\begin{array}{c}\text { Conventional } \\
\text { group } \\
(20)\end{array}$ & $\begin{array}{c}\text { Incremental } \\
\text { group } \\
(20)\end{array}$ & $\begin{array}{c}\text { Chi } \\
\text { square } \\
\text { test }\end{array}$ \\
\cline { 4 - 5 } & & $8(40 \%)$ & $2(10 \%)$ & P value \\
\hline Hypotension & $+\mathrm{ve}$ & $0.03^{*}$ \\
\hline $\begin{array}{c}\text { Hospital } \\
\text { admission }\end{array}$ & $+\mathrm{ve}$ & $6(30 \%)$ & $2(10 \%)$ & $=0.1185$ \\
\hline
\end{tabular}

Table 2: Comparison between the two groups with respect to the results data using the Chi square test:

Comparative among the two groups identified; substantial decrease in the incidence of hypotension in incremental groups $(40 \%)$; relative to conventional groups $(10 \%)$; with a very significant statistical difference $(\mathrm{p}=0.03)$ (Table 2, Fig. 5).

Non-significant differences in hospital admission were observed in the comparative analysis among the 2 groups ( $p>0.05)$. 


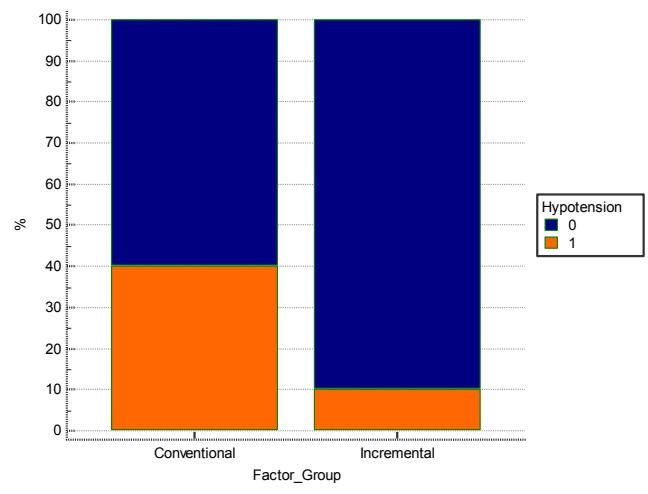

Fig.5: Comparison between the 2 groups of patients regarding hypotension.

\section{DISCUSSION}

This was a prospective comparative research performed in 40 patients with chronic kidney disease (CKD); to assess incremental hemodialysis (twice/week) compared with conventional dialysis (thrice/week) for the conservation of residual kidney function among patients initiating regular hemodialysis.

The study will be conducted in patients with chronic end stage kidney disease through 2018 and 2019 in Military hospitals (Maadi Military Hospital, kobrielkoba military hospital and air force military hospital). We assigned 40 patients with chronic kidney disease stage V D who early started hemodialysis.

Group1: 20 patients underwent incremental hemodialysis (twice/week) and Group 2: 20 patients underwent conventional hemodialysis (thrice/week).

Patients have been followed for laboratory findings of (complete blood picture, serum creatinine, urea, calcium, albumin, phosphorus, parathyroid hormone, alkaline phosphatase, glomerular filtration rate).

Regarding Baseline data, we found that; the mean age of all patients was $(45.95 \pm 2.97)$ years, and the average UOP was (1124.3 \pm 337.2) $\mathrm{ml} /$ day. Regarding gender of the patients, the majority $(60 \%)$ of patients were males; while $(40 \%)$ were females, with $(65 \%)$ had DM, and $(75 \%)$ had HTN, which came in agreement with A. Mathew et al. ${ }^{7}$ and Lucas et al. ${ }^{8}$.

Regarding Outcome data, (20\%) of patients had hypotension, (15\%) had hospital admission, while nobody suffered mortality, which came in agreement with Lucas et al. ${ }^{8}$

Lucas et al reported thatin the 2- and 3-HD / week groups, hospitalization period was 4 (0-15) and 11 (2-14) days/patient-years, respectively (median and IQR, $P=0,068){ }^{8}$

No substantial difference in the comparative study between the 2 groups with respect to all basic clinical data $(p>0.05)$, which came in agreement with A. Mathew et al. ${ }^{7}$, Lucas et al. ${ }^{8}$ and Liu et al . ${ }^{9}$

A. Mathew et al. reported that, no statistical disparity with all-cause mortality in subgroups of age, gender
, ethnicity, central venous catheter use or diabetes condition in preset subgroup analyses of the matched cohort compared incremental HD to conventional HD. ${ }^{7}$

We found marked increase in UOP in incremental group; compared to conventional group; during the serial measurements $(p<0.05)$, which came in agreement with Liu et al. ${ }^{9}$, A. Mathew et al. ${ }^{7}$, Lucas et al. ${ }^{8}$, A. T. Mathew et al. ${ }^{10}$ and Merino et al. ${ }^{11}$

Liu et alreported that those on incremental HD appeared to be female and thinner relative to those on traditional HD. The amount of urine in the incremental HD group was higher relative to the conventional HD group ${ }^{9}$

Lucas et alreported thatthe daily volume of urine and GFR (glomerular filtration rate) is higher throughout the $2 \mathrm{HD} /$ week group. In patients who began 2- and $3 \mathrm{HD} /$ week, the GFR range was $2-12,6 \mathrm{~mL} / \mathrm{min}$ and $0,2-13,6 \mathrm{~mL} / \mathrm{min}$, respectively. ${ }^{8}$

We found marked increase in hemoglobin in incremental group; compared to conventional group; during the serial measurements $(\mathrm{p}<0.05)$, which came in agreement with Merino et al. ${ }^{11}$ and A. Mathew et al.

Hemoglobin and hematocrit enhanced after 1 year and the mean dose of erythropoiesis stimulating factors (ESF) did not raise significantly, reported by Merino et al. 2017, which was darbepoetin ( $\mathrm{mcg} /$ week), $27 \pm 21$ and $30 \pm 22 \mathrm{mcg} /$ week, $\mathrm{p}=0.725$ in our case. ${ }^{11}$

Liu et alreported thatthose on incremental HD appeared to be female and thinner relative to patients on traditional HD. The calcium and hemoglobin levels between the groups were similar. ${ }^{9}$

Lucas et al reported thatboth groups had similar hemoglobin concentrations;however, the weekly erythropoietin dose was substantially lower in patients, who began $2 \mathrm{HD} /$ week ${ }^{8}$

We found marked increase in post-dialysis urea in incremental group; compared to conventional group; during the serial measurements $(\mathrm{p}<0.05)$, which came in agreement with A. Mathew et al. 2016. ${ }^{7}$

A.Mathew et al reported thatpatients with an incremental HD regimen and those with sufficient baseline RKF had substantially more conservation of renal clearance of urea in that study ?

We found marked increase in GFR in incremental group; compared to conventional group; during the serial measurements $(p<0.05)$, which came in agreement withKalantar-Zadeh et al. ${ }^{12}$, Bolasco et al. ${ }^{13}$, Rhee et al. ${ }^{14}$, Liu et al. ${ }^{9}$, A. Mathew et al. ${ }^{7}$,Lucas et al. ${ }^{8}$ and Obi et al. ${ }^{14}$

In reality, Bolasco et al recorded faster loss of RKF by twice-weekly or more regular hemodialysis as a negative predictive factor for both mortality and morbidity; however, nephrologists have long undervalued the clinical significance of RKF. ${ }^{13}$

A. Mathew et al reported that, a prospective study of 168 patients with HD incidence showed that in twiceweekly HD-initiated patients, the proportion of 
patients with RKF loss was substantially lower in twice-weekly HD-initiated patients relative to conventional thrice-week HD-initiated patients ${ }^{7}$.

Obi et al reported thatboth the clearance of renal urea and the amount of urine in incremental vs conventional regimens ( $\mathrm{P}, 0.001$ for both) displayed considerably slower decreases over time in this matched cohort. ${ }^{14}$

We found marked increase in calcium in incremental group; compared to conventional group; during the serial measurements $(p<0.05)$, which came in agreement with A. Mathew et al. ${ }^{7}$ and Obi et al. ${ }^{14}$

A. Mathew et al reported that, the final cohort of the study consisted of 87,718 patients from 1737 facilities, comprising 201 frequent ( $\$ 4$ times weekly) HD patients from 158 facilities and 682 incremental (twice weekly or less) HD patients from 444 facilities, with increased calcium level in incremental group $9.1 \pm 0.5^{7}$

Obi et al reported that, Trends in the selected parameters indicated lower dialysis frequency, shorter dialysis period, lower hemoglobin and corrected serum calcium concentrations in patients treated with an incremental regimen. ${ }^{14}$

Liu et al reported that, there are no statistically substantial variations in serum levels of calcium, phosphate, albumin, hemoglobin or hospitalization rates among groups. Serum calcium $(\mathrm{SMD}=-0.397$, 95\% CI: -0.523 to $-0.272, \mathrm{P}<0.001 ; \mathrm{I} 2=0 \%$ ) relative to laboratory values at the end of follow-up (subgroup 1). ${ }^{9}$

We found marked decrease in phosphorus in incremental group; compared to conventional group; during the serial measurements $(p<0.05)$, which came in agreement with A. Mathew et $\mathrm{al}^{7}$, Merino et $\mathrm{al}^{11}$ and Liu et al. ${ }^{9}$

A. Mathew et al reported that, the final cohort of the study consisted of 87,718 patients from 1737 facilities, comprising 201 frequent ( $\$ 4$ times weekly) HD patients from 158 facilities and 682 incremental (twice weekly or less) HD patients from 444 facilities, with decreased phosphorus in incremental group $4.3 \pm 1.0 .^{7}$

Merino et al reported thatafter 1 year, serum phosphate level control had improved, but the variations were not statistically relevant.$^{11}$

Liu et al reported that, Serum phosphate levels in the incremental HD group were lower, and serum albumin levels were higher ${ }^{9}$.

We found marked decrease in PTH in incremental group; compared to conventional group; during the serial measurements $(\mathrm{p}<0.05)$, which came in agreement with Merino et al. ${ }^{11}$

Merino et al reported thatafter 1 year, control of PTH levels improved, but the variations were not statistically relevant. ${ }^{11}$

We found marked decrease in albumin in incremental group; compared to conventional group; during the serial measurements $(p<0.05)$, which came in disagreement with Liu et al. 9 .
Liu et al reported that, Serum phosphate levels in the incremental HD group were lower, and serum albumin levels were higher. ${ }^{9}$

We found marked decrease in $\mathrm{Kt} / \mathrm{V}$ in incremental group; compared to conventional group; during the serial measurements $(\mathrm{p}<0.05)$, which came in agreement with Lucas et al. ${ }^{8}$

A comparative study among the 2 groups demonstrated; a substantial decrease in the incidence of hypotension in the incremental group (40\%); a highly substantial statistical difference $(\mathrm{p}=0.03)$, relative to the conventional group (10\%); which came in agreement with A. Mathew et al. ${ }^{7}$, Merino et al. ${ }^{11}$, Kalantar-Zadeh et al. ${ }^{12}$ and A. T. Mathew et al. $^{10}$

Merino et al reported that, the average baseline systolic blood pressure $(\mathrm{mmHg})$ was $145 \pm 12 \mathrm{mmHg}$ versus $151 \pm 14$ after 12 months $(p=0.165)$ and the average baseline diastolic blood pressure was $72 \pm 13$ versus $67 \pm 20$ at baseline and 1 year $(\mathrm{p}=0.243)$. Blood pressure was similar at baseline relative to 12 months. ${ }^{11}$

Comparative study among the two groups showed no substantial difference in hospital admission ( $\mathrm{p}>$ 0.05 ), which came in agreement with A.T. Mathew et al. ${ }^{10}$, Liu et al. ${ }^{9}$ and Lucas et al. ${ }^{8}$

A. T. Mathew et al reported that, more frequent HD decreased the composite endpoint of vascular access loss, repair, or access-related hospitalization in the analysis of the FHN study. ${ }^{10}$

Kalantar-Zadeh et alreported that, this incremental approach to hemodialysis offers a more gradual and tolerable transition to renal replacement treatment, possibly resulting in improved quality of life linked to health, longer conservation of residual kidney function, reduction of inflammatory and oxidative stress caused by hemodialysis, reduced rate of intradialytic hypotension,, decreased stimulating agent dose of erythropoiesis and decreased mortality and morbidity ${ }^{12}$

\section{CONCLUSION}

To conclude, our research investigates the correlation among the frequency of HD treatment and survival of patient. In line with recent literature, our results show that some selected patients with acceptable RKF, sufficient control of interdialytic gaining weight, and low or moderate burden of comorbid disease (CCI < 5) in an incident HD population, an HD incremental approach may be deemed as an appropriate alternative to conventional thrice-weekly HD.

\section{REFERENCES}

1. O’Hare, A.M., Wong, S.P., Margaret, K.Y., Wynar, B., Perkins, M., Liu, C.-F., et al. Trends in the timing and clinical context of maintenance dialysis initiation.Journal of the American Society of Nephrology. 2015; 26 (8), 1975-81.

2. Vilar, E., Wellsted, D., Chandna, S.M., Greenwood, R.N., and Farrington, K. Residual renal function improves outcome in incremental haemodialysis 
despite reduced dialysis dose. Nephrology Dialysis Transplantation. 2009; 24 (8), 2502-10.

3. Wong, J., Vilar, E., Davenport, A., and Farrington, K Incremental haemodialysis. Nephrology Dialysis Transplantation. 2015; 30 (10), 1639-48.

4. Kessler, M., Canaud, B., Pedrini, L.A., Tattersall, J., ter Wee, P.M., Vanholder, R., et al. European Best Practice Guidelines for Haemodialysis (part 1): Section II: Haemodialysis adequacy.Neprol Dial Transpl. 2002; 17 16-31.

5. Suri, R.S., Larive, B., Sherer, S., Eggers, P., Gassman, J., James, S.H., et al. Risk of vascular access complications with frequent hemodialysis. Journal of the American Society of Nephrology. 2013; 24 (3), 498-505.

6. Collins, A.J., Foley, R.N., Gilbertson, D.T., and Chen, S.-C. United States Renal Data System public health surveillance of chronic kidney disease and end-stage renal disease.Kidney International Supplements. 2015; 5 (1), 2-7.

7. Mathew, A., Obi, Y., Rhee, C.M., Chen, J.L., Shah, G., Lau, W.-L., et al. Treatment frequency and mortality among incident hemodialysis patients in the United States comparing incremental with standard and more frequent dialysis. Kidney International. 2016; 90 (5), 1071-9.

8. Lucas, M.F., Teruel, J.L., Ruíz-Roso, G., Díaz, M., Raoch, V., Caravaca, F., et al. Incremental hemodialysis schedule in patients with higher residual renal function at the start of dialysis. Advances in Nephrology. 2014.
9. Liu, Y., Zou, W., Wu, J., Liu, L., and He, Q. Comparison between incremental and thrice-weekly haemodialysis: Systematic review and meta-analysis. Nephrology. 2019; 24 (4), 438-44.

10. Mathew, A.T., Obi, Y., Rhee, C.M., Chou, J.A., and Kalantar-Zadeh, K. Incremental dialysis for preserving residual kidney function-Does one size fit all when initiating dialysis? in: Seminars in Dialysis, Wiley Online Library, pp. 2018; 343-52.

11. Merino, J.L., Domínguez, P., Bueno, B., Amézquita, Y., Espejo, B., and Paraíso, V. Application of model of incremental haemodialysis, based on residual renal function, at the initiation of renal replacement therapy. Nefrología (English Edition). 2017; 37 (1), $39-46$.

12. Kalantar-Zadeh, K., Crowley, S.T., Beddhu, S., Chen, J.L., Daugirdas, J.T., Goldfarb, D.S., et al. Renal replacement therapy and incremental hemodialysis for veterans with advanced chronic kidney disease. in: Seminars in Dialysis, Wiley Online Library, pp. 2017; 251-61.

13. Bolasco, P., Cupisti, A., Locatelli, F., Caria, S., and Kalantar-Zadeh, K. Dietary management of incremental transition to dialysis therapy: onceweekly hemodialysis combined with low-protein diet. Journal of Renal Nutrition. 2016; 26 (6), 352-9.

14. Obi, Y., Streja, E., Rhee, C.M., Ravel, V., Amin, A.N., Cupisti, A., et al. Incremental hemodialysis, residual kidney function, and mortality risk in incident dialysis patients: a cohort study. American Journal of Kidney Diseases. 2016; 68 (2), 256-5. 